\title{
DIFERENCIAÇÃO DA VEGETAÇÃO ARBÓREA DE TRÊS SETORES DE UM REMANESCENTE FLORESTAL RELACIONADA AO SEU HISTÓRICO DE PERTURBAÇÕES
}

\author{
DIFFERENTIATION OF ARBOREAL VEGETATION IN THREE SECTORS OF A FOREST \\ FRAGMENT RELATED TO ITS HISTORICAL DISTURBANCES
}

\author{
Jean Daniel Morel ${ }^{1}$ José Aldo Alves Pereira ${ }^{2}$ Rubens Manoel dos Santos ${ }^{3}$ \\ Evandro Luiz Mendonça Machado ${ }^{4}$ João José Marques ${ }^{5}$
}

\begin{abstract}
RESUMO
A fragmentação florestal é um dos principais agentes de redução da biodiversidade, interferindo diretamente nos processos ecológicos da comunidade arbórea. Este trabalho teve como objetivo avaliar parâmetros de diversidade, estrutura e composição em três setores de um remanescente florestal com históricos distintos de perturbações. A vegetação arbórea foi avaliada em 24 parcelas de $10 \times 10 \mathrm{~m}$, amostrando um total de 1228 indivíduos vivos. Foram quantificados o índice de diversidade de Shannon, a equabilidade de Pielou e os estimadores jackknife de primeira e segunda ordem. Os indivíduos amostrados foram distribuídos em classes de diâmetro e o valor de importância (VI) foi calculado para as espécies. Foi também realizada uma Análise de Correspondência Retificada (DCA) para verificar se há distinção entre os três setores. Constatou-se que o setor onde ocorreu corte raso e queima da vegetação possui maior abundância e riqueza, porém, tem a pior equabilidade, o que condiz com os efeitos das perturbações, corroborado também pela distribuição nas classes diamétricas e pelas espécies de maior VI. O setor que não possui perturbações e que está situado em um local com maior variedade de ambientes apresentou diversidade, estrutura e composição condizentes com estas situações. Já o outro setor, que não sofreu corte raso, está submetido ao pisoteio pelo gado e é ambientalmente semelhante ao primeiro setor, apresenta parâmetros condizentes com a ausência de perturbações severas. Por outro lado, a menor diversidade ambiental também o diferencia, o que o coloca em uma situação intermediária.
\end{abstract}

Palavras-chave: ecologia florestal; impactos ambientais; Floresta Estacional Semidecidual.

\begin{abstract}
Forest fragmentation is one of the main causes of biodiversity loss, directly affecting the ecological processes. This study aimed to evaluate tree diversity, structure, and composition parameters in three sectors of a forest fragment with distinct disturbance records. The arboreal vegetation was evaluated in twenty-four $10 \times 10$ $\mathrm{m}$ plots, sampling a total of 1,228 living individuals. We calculated Shanon's diversity index, Pielou's equability, and jackknife estimators of first and second orders. The sampled individuals were distributed in diameter classes and the importance value (VI) was calculated for each species. It was made a Detrended
\end{abstract}

1 Engenheiro Florestal, Dr., Pós-doutorando no Departamento de Ciências Florestais, Universidade Federal de Lavras, Campus Universitário, Caixa Postal 3037, CEP 37200-000, Lavras (MG), Brasil. morel.jean@gmail.com

2 Engenheiro Florestal, PhD., Professor Associado do Departamento de Ciências Florestais, Universidade Federal de Lavras, Campus Universitário, Caixa Postal 3037, CEP 37200-000, Lavras (MG), Brasil. j.aldo@dcf.ufla.br

3 Biólogo, Dr., Professor Adjunto do Departamento de Ciências Florestais, Universidade Federal de Lavras, Campus Universitário, Caixa Postal 3037, CEP 37200-000, Lavras (MG), Brasil. rubensmanoel@dcf.ufla.br

4 Engenheiro Florestal, Dr., Professor Adjunto do Departamento de Engenharia Florestal, Faculdade de Ciências Florestais, Universidade Federal dos Vales do Jequitinhonha e Mucuri, Rod. MGT 367 - Km 583, 5000, CEP 39100-000, Diamantina (MG), Brasil.machadoelm@gmail.com

5 Engenheiro Agrônomo, PhD., Professor Associado do Departamento de Ciência do Solo, Universidade Federal de Lavras, Campus Universitário, Caixa Postal 3037, CEP 37200-000, Lavras (MG), Brasil. jmarques@dcs.ufla.br

Recebido para publicação em 18/02/2013 e aceito em 5/02/2014

Ci. Fl., v. 26, n. 1, jan.-mar., 2016 
Correspondence Analysis (DCA) to verify whether there were significant distinctions between the sectors. It was noticed that the sector where there was clear cutting and vegetation burning in a recent past had higher abundance and richness but also the worst equability. That corresponds to the effects of perturbation as confirmed by the tree diameters and the presence of species of greater importance value. The sector that had no record of disturbance, situated in a location with greater variety of microenvironments, presented diversity, structure, and composition consistent with a no disturbance scenario. The other sector, which did not have clear cutting, was subjected to cattle trampling presented ecological parameters consistent with the absence of major disturbances. On the other hand, this third sector had the smallest environmental diversity, which puts this last sector in an intermediate situation.

Keywords: forest ecology, environmental impacts, Seasonal Semidecidual Forest.

\section{INTRODUÇÃO}

A maior parte dos problemas ambientais que provocam mudanças na distribuição e abundância dos organismos é decorrente da fragmentação antrópica, que é considerada o principal agente de redução da biodiversidade (PEREIRA; OLIVEIRA FILHO; LEMOS FILHO, 2007; LAURANCE et al., 2011; HORNER et al., 2012). Sabe-se que, ao longo da sucessão, os processos ecológicos em florestas secundárias são fortemente influenciados por fatores de distúrbio, como frequência de queimadas, pastejo e outros fatores antrópicos (MARTINEZGARZA; HOWE, 2003; HORNER et al., 2012). Neste processo, a velocidade de regeneração muitas vezes é determinada pela intensidade dos distúrbios, atuando sobre a diversidade e estrutura da comunidade vegetal (CANTARELLO et al, 2011; HORNER et al., 2012, IMAI et al., 2012).

A região do Alto Rio Grande, que compõe o Domínio Atlântico, possui um histórico de perturbações antrópicas que remonta aos primórdios da colonização portuguesa em Minas Gerais, em fins do século XVII, advindo das atividades de mineração, agricultura e pecuária (DEAN, 1996; VILELA, 2007). Nesta região, a cobertura florestal está reduzida a fragmentos esparsos, em sua maior parte com menos de 10 ha, visto que a ocorrência da vegetação arbórea coincidia com os solos mais férteis e úmidos e, portanto, mais visados pela agropecuária (OLIVEIRA FILHO; SCOLFORO; MELLO, 1994; OLIVEIRA FILHO; MELLO; SCOLFORO, 1997).

Diversos estudos foram realizados nos remanescentes florestais do Alto Rio Grande e alguns relacionaram as características da vegetação com impactos de origem antrópica (OLIVEIRA FILHO; SCOLFORO; MELLO, 1994; OLIVEIRA FILHO; MELLO; SCOLFORO, 1997; NUNES et al., 2003; PEREIRA; OLIVEIRA FILHO; LEMOS
FILHO, 2007; MACHADO et al., 2008). Estudos deste tipo permitem compreender como a vegetação se comporta quando sujeita a diferentes formas de perturbações. Entretanto, na maioria das vezes têm-se poucas informações sobre o histórico das áreas, o que dificulta inferir sobre o status atual da vegetação em relação a parâmetros ecológicos e conservacionistas.

Partindo da assertiva de que a comparação de parâmetros fitossociológicos entre comunidades arbóreas fornece importantes respostas sobre a influência de distúrbios antrópicos (IMAI et al., 2012), este trabalho teve como objetivo comparar três setores de um remanescente de Floresta Estacional Semidecidual, situado no Município de Itumirim, Minas Gerais, através da composição e estrutura da vegetação arbórea. Pela análise dos dados buscou-se verificar se os setores diferem entre si, relacionando as características da vegetação ao seu histórico de perturbações.

\section{MATERIAL E MÉTODOS}

\section{Caracterização da área de estudo}

A área de estudo está situada próxima às coordenadas geográficas $21^{\circ} 16^{\prime} 35^{\prime} \% \mathrm{~S}$ e 4449'34”'W, no Município de Itumirim, localizado no alto Rio Grande, sul de Minas Gerais. O clima é do tipo Cwa, caracterizado por invernos secos e verões chuvosos. A temperatura média anual é de $19,4^{\circ} \mathrm{C}$ (média de $15,8^{\circ} \mathrm{C}$ em julho e $22,1^{\circ} \mathrm{C}$ em fevereiro) e a precipitação normal anual é de $1530 \mathrm{~mm}$, sendo julho o mês mais seco (23 mm) e dezembro o mês mais chuvoso (296 mm) (BRASIL, 1992).

$\mathrm{O}$ trabalho foi conduzido em um remanescente florestal com cerca de 10 ha, com altitude variando entre 920 e 956 m. A vegetação é classificada como Floresta Estacional Semidecidual Montana (VELOSO; RANGEL FIHO; LIMA, 
1991). Para efeito de comparação, o remanescente foi considerado em três setores: o setor A, que possui 2,5 ha e está situado em uma vertente pouco inclinada, a oeste; o setor B, com 3 ha e nas mesmas condições de relevo, situado na vertente oposta; e o setor $\mathrm{C}$, com 3 ha, que está situado em uma área de relevo acidentado, ao sul dos demais setores, e onde estão localizadas as nascentes do curso de água que separa os setores A e B. Quanto aos tipos de solo (EMBRAPA, 2006), no setor A predomina o Latossolo Vermelho Amarelo distrófico típico, mesmo tipo predominante no setor B. Já no setor $\mathrm{C}$ predominam o Cambissolo Háplico Tb distrófico típico e o Neossolo Flúvico Tb distrófico típico.

De acordo com informações dos moradores locais, o setor A foi submetido a corte raso, seguido de queima em 1965. Após um ano de cultivo, a área foi convertida em pastagem, que foi abandonada depois do segundo ano. A vegetação que se estabeleceu passou a sofrer cortes seletivos de madeira e a ser esporadicamente acessada pelo gado. O setor B anteriormente era conectado a outro remanescente, situado na mesma vertente. Em 1965, a área, hoje existente entre esse remanescente e o setor $\mathrm{B}$, foi submetida a corte raso. Nessa área, houve alternância entre cultivos e pastagem, sendo que na maior parte dos anos o gado teve acesso ao setor. No entanto, o local em que foi feita a amostragem nunca sofreu corte raso e mesmo a extração seletiva de madeira, quando ocorreu, foi feita com pouca frequência. Já sobre o setor $C$, as informações são de que este nunca sofreu intervenções antrópicas.

\section{Coleta e análise dos dados}

Foram locadas, em cada setor, 24 parcelas de $10 \times 10 \mathrm{~m}$, com $4 \mathrm{~m}$ de distância entre si. Dentro delas foram medidos os diâmetros a 1,3 m de altura (DAP) de todos os indivíduos arbóreos vivos que apresentaram medida igual ou superior a $5 \mathrm{~cm}$. A identificação botânica seguiu os critérios do Angiosperm Phylogeny Group (APG, 2003). A normalidade dos dados foi verificada pelo teste D'Agostino-Pearson. Em seguida foram realizadas análises de variância (ANOVA) e testes Tukey (ZAR, 1996). Estas análises foram feitas no programa BioEstat 5.0. Foram calculados o índice de diversidade de Shannon $\left(H^{\prime}\right)$, a equabilidade de Pielou ( $\left.J^{\prime}\right)$ (BROWER; ZAR, 1984) e os estimadores jackknife de primeira e segunda ordem, os quais estimam o número total de espécies a partir da heterogeneidade da amostra (PALMER, 1991).
Os índices de Shannon foram comparados pelo teste $\mathrm{t}$ de Hutchenson. Os indivíduos amostrados foram distribuídos em classes de diâmetro, empregando intervalos com amplitudes crescentes para compensar o decréscimo da densidade nas maiores classes (BOTREL et al., 2002). Foi também calculado o valor de importância (VI) das espécies, que é dado pela soma da densidade, frequência e dominância, em termos relativos (MUELLERDOMBOIS; ELLENBERG, 1974). Com os VI foram elaborados ranks para as espécies, com o fim de identificar as mais importantes em cada setor. Para verificar se os três setores se distinguem em função da distribuição das espécies nas parcelas foi feita uma Análise de Correspondência Retificada (DCA) (BRAAK, 1987). Para isso, utilizou-se uma matriz com a abundância das espécies e outra contendo uma variável categórica correspondente ao setor de cada parcela. Como recomendado por Braak (1995), os dados de abundância foram logaritmizados ( $\ln$ (x $+1)$ ) para compensar os desvios provocados por valores muito discrepantes.

\section{RESULTADOS E DISCUSSÃO}

Foram amostrados 1228 indivíduos arbóreos, sendo 533 no setor A, 358 no setor B e 337 no setor C. Os indivíduos foram classificados em 154 espécies pertencentes a 55 famílias. O setor A apresentou 37 famílias e 95 espécies; o setor B, 33 famílias e 79 espécies; e o setor C, 39 famílias e 88 espécies. Estes valores mostraram diferenças significativas entre os setores pela análise de variância. Entretanto, o teste Tukey indicou que não há diferenças significativas entre as médias quando comparados os setores B e C (Tabela 1).

A abundância do setor A representa 43,4\% do total de indivíduos amostrados. O setor foi também o mais rico em espécies e famílias, o que provavelmente se deve ao corte raso seguido da queima, realizado há mais de 40 anos. Sabe-se que este tipo de perturbação estimula o crescimento vegetal, iniciando o período sucessional e, com o passar do tempo, as espécies lumínicas passam a coexistir com as que se estabelecem sob elas, o que proporciona maior abundância (CONNELL; SLATYER, 1977; OLIVEIRA FILHO; MELLO; SCALFORO, 1997; TABARELLI; MANTOVANI, 1999; OMEJA et al., 2011; IMAI et al., 2012). O fato dos setores $\mathrm{B}$ e $\mathrm{C}$ não terem diferido significativamente entre si pode ser devido à ausência de corte raso, já que valores equivalentes de abundância podem ocorrer 
TABELA 1: Médias de indivíduos, famílias e espécies arbóreas por parcela e índices de riqueza e diversidade para três setores de um remanescente florestal situado no Município de Itumirim MG. Médias seguidas por letras diferentes indicam valores não significativos entre os setores pelos testes Tukey e $t$ de Hutchenson.

TABLE 1: Average measurements for individuals, families, and tree species for plots and indices of richness and diversity in three sectors of a forest fragment located in Itumirim, Minas Gerais state, Brazil. Means followed by same letters indicate no significant differences among sectors by Tukey and $\mathrm{t}-\mathrm{Hutchenson}$ tests.

\begin{tabular}{|c|c|c|c|c|c|c|c|c|c|}
\hline \multicolumn{6}{|c|}{ Abundância } & \multicolumn{4}{|c|}{ Diversidade } \\
\hline & Setor & Média & & $F$ & $p$ & Índice & Setor & Valor & \\
\hline & A & 22,21 & $\mathrm{a}$ & & & & A & 3,84 & $a b c$ \\
\hline \multirow[t]{3}{*}{ Indivíduos } & B & 14,92 & $\mathrm{bc}$ & 17,1021 & $<0,0001$ & Índice de Shannon (H') & B & 3,76 & $a b$ \\
\hline & $\mathrm{C}$ & 14,04 & $\mathrm{bc}$ & & & & $\mathrm{C}$ & 3,93 & $\mathrm{ac}$ \\
\hline & A & 11,42 & $\mathrm{a}$ & & & & A & 0,84 & \\
\hline \multirow[t]{3}{*}{ Famílias } & B & 8,54 & $\mathrm{bc}$ & 13,6251 & $<0,0001$ & Equabilidade de Pielou (J') & B & 0,86 & \\
\hline & $\mathrm{C}$ & 8,50 & $\mathrm{bc}$ & & & & $\mathrm{C}$ & 0,88 & \\
\hline & A & 14,38 & $\mathrm{a}$ & & & & A & 132,40 & \\
\hline \multirow[t]{5}{*}{ Espécies } & B & 10,13 & $\mathrm{bc}$ & 18,4088 & $<0,0001$ & Estimador jackknife; $1^{\mathrm{a}}$ ordem & B & 107,70 & \\
\hline & $\mathrm{C}$ & 10,29 & $\mathrm{bc}$ & & & & $\mathrm{C}$ & 128,20 & \\
\hline & & & & & & & A & 158,50 & \\
\hline & & & & & & Estimador jackknife; $2^{\mathrm{a}}$ ordem & B & 117,70 & \\
\hline & & & & & & & $\mathrm{C}$ & 153,60 & \\
\hline
\end{tabular}

em áreas com condições sucessionais semelhantes (UHL; MURPHY, 1981; WILLIAMS-LINERA; LOREA, 2009; MAGNAGO et al., 2011; IMAI et al., 2012).

A diversidade de espécies, avaliada pelo índice de Shannon, variou de 3,76 nats/indivíduo no setor B a 3,93 nats/indivíduo no setor C (Tabela 1). De acordo com o teste $t$ de Hutchenson, não houve diferença significativa entre os setores A e B e entre A e C. A equabilidade de Pielou variou de 0,84 no setor A a 0,88 no setor C. Os estimadores jackknife indicaram maior riqueza potencial para o setor A, seguido pelo setor C. Avaliando o setor C, sua maior diversidade provavelmentese deveàheterogeneidade ambiental, com variação de solos e relevo, uma vez que cada combinação de variáveis ambientais pode corresponder a diferentes dominâncias ecológicas (TERBORGH, 1992; CARVALHO et al., 1995; RODRIGUES et al., 2003; HORNER et al., 2012). Este setor também apresentou maior equabilidade, indicando melhor distribuição dos indivíduos nas espécies. O fato da diversidade no setor $A$ não diferir significativamente da apresentada pelo setor $\mathrm{C}$, que possui a maior diversidade, e de $\mathrm{B}$, que possui a menor, deve-se à ocorrência simultânea de espécies com capacidade de colonizar diferentes ambientes e que normalmente ocorrem em estágios iniciais e intermediários de regeneração (TABARELLI; MANTOVANI, 1999; NUNES et al., 2003; OMEJA et al., 2011; IMAI et al., 2012). A equabilidade do setor A também mostra que, dentre os três setores, ele é o que possui a pior distribuição de indivíduos nas espécies, indicando que ainda não houve pressão seletiva suficiente para proporcionar exclusão (OLIVEIRA FILHO et al., 2004). Quando comparados os estimadores de riqueza jackknife, os maiores valores do setor A também sugerem uma condição sucessional mais baixa, mostrando que há um maior acúmulo de espécies em função de sua área. O setor $\mathrm{A}$ é seguido pelo $\mathrm{C}$, que apresenta maior heterogeneidade ambiental. Já o setor B possui valores de riqueza característicos de floresta madura, provavelmente devido à ausência de corte raso, o que está de acordo com a literatura que afirma que a riqueza e a diversidade de espécies em florestas tropicais frequentemente são menores em florestas maduras que naquelas que sofrem distúrbios intensos (BROWN; LUGO, 1990; TABARELLI; MANTOVANI, 1999; IMAI et al., 2012). Assim, a menor riqueza do setor $\mathrm{B}$ em relação ao setor $\mathrm{C}$ pode ser justificada pela ausência de influência ripária e relevo movimentado, fatores que condicionam maior 
diversidade de habitat, implicando em maior riqueza de espécies (TERBORGH, 1992; RODRIGUES et al., 2003; JONES; SZYSKA; KESSLER 2011; GUILHERME et al., 2012; SCIPIONI et al., 2012). Os impactos decorrentes do pisoteio pelo gado também podem ter atuado na redução da diversidade do setor B, visto que este tipo de perturbação pode alterar a composição e a estrutura da vegetação em florestas tropicais (WILLIAMS-LINERA; LOREA, 2009; CANTARELLO et al., 2011; HORNER et al., 2012).

Quanto à distribuição diamétrica, o setor $\mathrm{A}$ apresentou maior abundância nas classes $5<10 \mathrm{~cm}$ e $10<20 \mathrm{~cm}$. Este setor foi superado por C e B na classe $20<40 \mathrm{~cm}$ e por B e C na classe $40<80$ $\mathrm{cm}$ (Figura 1). A maior densidade de indivíduos nas menores classes está de acordo com as afirmações de que florestas em estágios menos avançados de regeneração possuem alto número de indivíduos com menores diâmetros (UHL; MURPHY, 1981; TABARELLI; MANTOVANI, 1999; CARVALHO; NASCIMENTO, 2009; MAGNAGO et al., 2011; FINOTTI et al., 2012). Também cabe observar as menores densidades nas classes dos setores B e C (Tabela 2), que não sofreram perturbação por corte raso, já que a redução na densidade total de indivíduos é considerada típica com o avanço sucessional (BROWN; LUGO, 1990; TABARELLI; MANTOVANI, 1999, CANTARELLO et al., 2011; MAGNAGO et al., 2011; IMAI et al., 2012).

Com relação ao VI, têm-se no setor A Copaifera langsdorffi, Cordia sellowiana e Cupania vernalis como as espécies mais importantes. No setor B, estas foram Copaifera langsdorffii, Trichilia catigua e Cheiloclinium cognatum. Já no setor C,

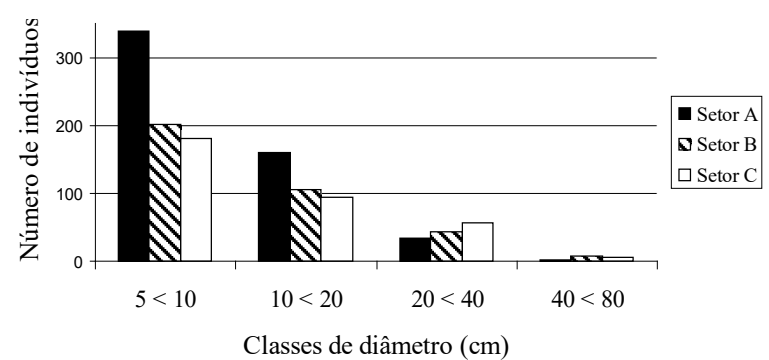

FIGURA 1: Distribuição em classes diamétricas dos indivíduos arbóreos em três setores de um remanescente florestal situado no Município de Itumirim MG.

FIGURE 1: Diameter class distribution of arboreal individuals in three sectors of a forest fragment located in Itumirim, Minas Gerais state, Brazil.

Copaifera langsdorffii, Naucleopsis oblongifolia e Protium spruceanum foram as que apresentaram os maiores VI (Tabela 3).

Copaifera langsdorffii, espécie com maior VI nos três setores, é dominante na maioria dos remanescentes florestais do centro-sul de Minas Gerais (OLIVEIRA FILHO; SCOLFORO; MELLO, 1994). Outras espécies que apresentaram altos VI neste trabalho também se mostraram importantes na estrutura de outros fragmentos da região, como Cupania vernalis, Ocotea odorifera, Protium widgrenii e Tapirira obtusa (OLIVEIRA FILHO; SCOLFORO; MELLO, 1994; CARVALHO et al., 1995; MACHADO et al.,

TABELA 2: Médias e desvios-padrão para densidade de indivíduos por parcela em três setores de um remanescente florestal situado no Município de Itumirim - MG. Valores de $p<0,05$ indicam diferenças significativas entre os setores.

TABLE 2: Means and standard deviations for density of individuals in 24 plots from three sectors of a forest fragment located in Itumirim, Minas Gerais state, Brazil. Values of $p<0.05$ indicate significant differences between sectors.

\begin{tabular}{cccccccccccccc}
\hline Classe diamétrica & \multicolumn{3}{c}{ Setor A } & \multicolumn{4}{c}{ Setor B } & \multicolumn{3}{c}{ Setor C } & \multicolumn{2}{c}{ F } & $p$ \\
\hline $5<80 \mathrm{~cm}$ & 22,21 & \pm & 6,69 & 14,92 & \pm & 4,17 & 14,04 & \pm & 4,75 & 17,102 & $<0,0001$ \\
$5<10 \mathrm{~cm}$ & 13,74 & \pm & 3,82 & 8,26 & \pm & 3,09 & 7,65 & \pm & 2,98 & 25,573 & $<0,0001$ \\
$10<20 \mathrm{~cm}$ & 6,95 & \pm & 3,70 & 4,55 & \pm & 2,30 & 4,09 & \pm & 2,48 & 5,774 & 0,0051 \\
$20<40 \mathrm{~cm}$ & 1,94 & \pm & 1,03 & 2,22 & \pm & 1,00 & 2,41 & \pm & 1,18 & 3,511 & 0,0343 \\
$40<80 \mathrm{~cm}$ & 1,00 & & & 1,14 & \pm & 0,38 & 1,20 & \pm & 0,45 & - & - \\
\hline
\end{tabular}


TABELA 3: Lista de espécies arbóreas amostradas nos três setores de um remanescente florestal situado no Município de Itumirim - MG, em que: VI = valor de importância; e Rank = classificação das espécies em função do VI.

TABLE 3: List of tree species sampled in three sectors of a forest fragment located in Itumirim, Minas Gerais state, Brazil, where: VI = importance value; and Rank = VI species classification.

\begin{tabular}{|c|c|c|c|c|c|c|c|c|}
\hline \multirow{3}{*}{$\begin{array}{ll} & \text { Família / Espécie } \\
\text { Anacardiaceae } & \end{array}$} & \multicolumn{8}{|c|}{ Setores } \\
\hline & \multicolumn{2}{|c|}{$\mathrm{A}$} & \multicolumn{2}{|c|}{$\mathrm{B}$} & \multicolumn{2}{|c|}{$\mathrm{C}$} & \multicolumn{2}{|c|}{ Total } \\
\hline & VI & Rank & VI & Rank & VI & Rank & VI & Rank \\
\hline Tapirira guianensis Aubl. & 2,65 & 37 & 0,00 & & 1,74 & 45 & 1,51 & 60 \\
\hline Tapirira obtusa (Benth.) J.D. Mitch. & 9,59 & 6 & 10,25 & 9 & 11,92 & 4 & 10,71 & 3 \\
\hline \multicolumn{9}{|l|}{ Annonaceae } \\
\hline Annona cacans Warm. & 2,73 & 36 & 0,00 & & 2,03 & 38 & 1,73 & 51 \\
\hline Duguetia lanceolata A.St.-Hil. & 7,18 & 9 & 2,74 & 31 & 2,56 & 30 & 4,42 & 18 \\
\hline Guatteria australis A.St.-Hil. & 1,06 & 61 & 1,52 & 55 & 0,78 & 74 & 1,11 & 79 \\
\hline Rollinia laurifolia Schltdl. & 4,32 & 23 & 1,85 & 49 & 2,35 & 32 & 2,85 & 23 \\
\hline Xylopia brasiliensis Spreng. & 0,00 & & 1,01 & 62 & 4,63 & 18 & 1,69 & 55 \\
\hline \multicolumn{9}{|l|}{ Aquifoliaceae } \\
\hline Ilex cerasifolia Reissek & 2,41 & 39 & 0,75 & 75 & 0,00 & & 1,18 & 74 \\
\hline Ilex conocarpa Reissek & 0,52 & 94 & 0,00 & & 0,00 & & 0,21 & 153 \\
\hline \multicolumn{9}{|l|}{ Araliaceae } \\
\hline $\begin{array}{l}\text { Schefflera calva (Cham.) Frodin \& Fiaschi } \\
\text { Arecaceae }\end{array}$ & 0,00 & & 0,00 & & 3,69 & 26 & 1,12 & 78 \\
\hline Euterpe edulis Mart. & 0,00 & & 0,00 & & 5,49 & 15 & 1,62 & 57 \\
\hline \multicolumn{8}{|l|}{ Asteraceae } & 29 \\
\hline \multicolumn{8}{|l|}{ Bignoniaceae } & 53 \\
\hline \multicolumn{9}{|l|}{ Boraginaceae } \\
\hline \multicolumn{9}{|l|}{ Burseraceae } \\
\hline Protium heptaphyllum (Aubl.) Marchand & 0,81 & 68 & 0,90 & 64 & 9,96 & 7 & 3,64 & 21 \\
\hline Protium spruceanum (Benth.) Engl. & 12,27 & 4 & 12,18 & 8 & 16,94 & 3 & 13,60 & 2 \\
\hline Protium widgrenii Engl. & 4,57 & 21 & 0,90 & 65 & 8,54 & 10 & 4,73 & 17 \\
\hline \multicolumn{9}{|l|}{ Canellaceae } \\
\hline Capsicodendron dinisii (Schwacke) Occhioni & 0,00 & & 0,00 & & 0,74 & 85 & 0,21 & 148 \\
\hline \multicolumn{9}{|l|}{ Celastraceae } \\
\hline Cheiloclinium cognatum (Miers.) A.C.Sm. & 0,53 & 87 & 17,00 & 3 & 0,00 & & 5,46 & 13 \\
\hline Maytenus salicifolia Reissek & 0,00 & & 0,00 & & 0,82 & 65 & 0,25 & 123 \\
\hline Salacia elliptica (Mart. ex Schult.) G.Don & 2,38 & 40 & 2,66 & 33 & 0,00 & & 1,72 & 52 \\
\hline \multicolumn{9}{|l|}{ Chrysobalanaceae } \\
\hline \multicolumn{9}{|l|}{ Clethraceae } \\
\hline \multicolumn{9}{|l|}{ Clusiaceae } \\
\hline Calophyllum brasiliense Cambess. & 0,00 & & 0,00 & & 7,57 & 12 & 2,36 & 35 \\
\hline Chrysochlamys saldanhae (Engl.) Oliveira-Filho & 0,00 & & 0,00 & & 1,17 & 59 & 0,37 & 114 \\
\hline Garcinia brasiliensis Mart. & 0,00 & & 0,00 & & 4,05 & 20 & 1,18 & 75 \\
\hline Terminalia glabrescens Mart. & 3,89 & 25 & 0,00 & & 0,00 & & 1,51 & 61 \\
\hline \multicolumn{9}{|l|}{ Cunoniaceae } \\
\hline Lamanonia grandistipularis (Taub.) Taub. & 0,00 & & 0,00 & & 1,82 & 40 & 0,60 & 98 \\
\hline Lamanonia ternata Vell. & 0,00 & & 0,00 & & 2,24 & 35 & 0,70 & 90 \\
\hline \multicolumn{9}{|l|}{ Cyatheaceae } \\
\hline Cyathea delgadii Sternb. & 0,00 & & 0,00 & & 1,53 & 52 & 0,45 & 111 \\
\hline \multicolumn{9}{|l|}{ Dicksoniaceae } \\
\hline Dicksonia sellowiana Hook. & 0,00 & & 0,00 & & 4,82 & 17 & 1,42 & 64 \\
\hline Elaeocarpaceae & & & & & & & & \\
\hline Sloanea monosperma Vell. & 0,53 & 84 & 0,00 & & 1,21 & 58 & 0,60 & 96 \\
\hline $\begin{array}{l}\text { Erythroxylaceae } \\
\text { Erythroxylon deciduum Saint-Hilaire }\end{array}$ & 0,54 & 80 & 0,00 & & 0,00 & & 0,22 & 138 \\
\hline
\end{tabular}

Continua... 
TABELA 3: Continuação...

TABLE 3: Continued...

\begin{tabular}{|c|c|c|c|c|c|c|c|c|}
\hline \multirow{2}{*}{ Família / Espécie } & \multicolumn{8}{|c|}{ Setores } \\
\hline & \multicolumn{2}{|c|}{ A } & \multicolumn{2}{|c|}{ B } & \multicolumn{2}{|c|}{$\mathrm{C}$} & \multicolumn{2}{|c|}{ Total } \\
\hline $\begin{array}{l}\text { Alchornea triplinervia (Spreng.) Müll.Arg. } \\
\text { Croton floribundus Spreng. } \\
\text { Pera glabrata (Schott) Poepp. ex Baill. } \\
\text { Sebastiania klotzschiana (Müll.Arg.) Müll.Arg. }\end{array}$ & $\begin{array}{l}0,00 \\
3,32 \\
7,61 \\
0,00\end{array}$ & $\begin{array}{c}28 \\
8\end{array}$ & $\begin{array}{c}2,70 \\
0,00 \\
14,93 \\
5,86 \\
\end{array}$ & $\begin{array}{c}32 \\
6 \\
12 \\
\end{array}$ & $\begin{array}{l}5,14 \\
0,00 \\
1,00 \\
0,00\end{array}$ & 60 & $\begin{array}{l}2,53 \\
1,20 \\
7,89 \\
1,74 \\
\end{array}$ & $\begin{array}{c}32 \\
73 \\
7 \\
50\end{array}$ \\
\hline $\begin{array}{l}\text { Fabaceae } \\
\text { Andira fraxinifolia } \text { Benth. } \\
\text { Bowdichia virgilioides Kunth } \\
\text { Copaifera langsdorffi Desf. } \\
\text { Copaifera trapezifolia Hayne } \\
\text { Dalbergia villosa (Benth.) Benth. } \\
\text { Hymenaea courbaril L. } \\
\text { Leucochloron incuriale (Vell.) Barneby \& J.W.Grimes } \\
\text { Machaerium brasiliense Vogel } \\
\text { Machaerium hirtum (Vell.) Stellfeld } \\
\text { Machaerium nictitans (Vell.) Benth. } \\
\text { Machaerium villosum Vogel } \\
\text { Ormosia arborea (Vell.) Harms } \\
\text { Platypodium elegans Vogel } \\
\text { Swartzia flaemingii Vogel } \\
\text { Tachigali rugosa (Mart. ex Benth.) Zarucchi \& Pipoly }\end{array}$ & $\begin{array}{c}2,28 \\
4,10 \\
27,77 \\
0,00 \\
3,26 \\
1,14 \\
4,77 \\
0,00 \\
0,85 \\
3,41 \\
6,00 \\
0,00 \\
4,84 \\
1,10 \\
2,62\end{array}$ & $\begin{array}{c}41 \\
24 \\
1 \\
\\
29 \\
57 \\
18 \\
\\
66 \\
27 \\
11 \\
\\
16 \\
58 \\
38\end{array}$ & $\begin{array}{c}2,49 \\
1,40 \\
17,65 \\
0,81 \\
0,78 \\
3,17 \\
0,00 \\
0,77 \\
0,00 \\
0,00 \\
1,03 \\
0,00 \\
0,00 \\
0,00 \\
3,14\end{array}$ & $\begin{array}{c}35 \\
57 \\
1 \\
69 \\
70 \\
25 \\
\\
72\end{array}$ & $\begin{array}{c}0,00 \\
0,00 \\
25,09 \\
5,89 \\
0,00 \\
0,00 \\
0,80 \\
0,00 \\
0,00 \\
0,00 \\
0,00 \\
0,78 \\
0,00 \\
0,00 \\
0,82\end{array}$ & $\begin{array}{c}1 \\
14\end{array}$ & $\begin{array}{c}1,66 \\
1,99 \\
24,59 \\
2,07 \\
1,41 \\
1,47 \\
1,93 \\
0,23 \\
0,31 \\
1,20 \\
2,54 \\
0,23 \\
1,88 \\
0,45 \\
2,06\end{array}$ & $\begin{array}{c}56 \\
43 \\
1 \\
41 \\
66 \\
62 \\
45 \\
131 \\
119 \\
72 \\
30 \\
128 \\
46 \\
110 \\
42\end{array}$ \\
\hline $\begin{array}{l}\text { Humiriaceae } \\
\text { Sacoglottis mattogrossensis Malme } \\
\text { Vantanea compacta (Schnizl.) Cuatrec. }\end{array}$ & $\begin{array}{l}0,86 \\
0,00\end{array}$ & 65 & $\begin{array}{l}0,00 \\
1,67\end{array}$ & 53 & $\begin{array}{l}0,74 \\
1,81 \\
\end{array}$ & $\begin{array}{l}87 \\
42\end{array}$ & $\begin{array}{l}0,53 \\
1,10\end{array}$ & $\begin{array}{c}102 \\
81\end{array}$ \\
\hline $\begin{array}{l}\text { Hypericaceae } \\
\text { Vismia guianensis (Aubl.) Pers. }\end{array}$ & 0,00 & & 0,77 & 71 & 0,00 & & 0,23 & 130 \\
\hline $\begin{array}{l}\text { Lacistemataceae } \\
\text { Lacistema hasslerianum Chodat }\end{array}$ & 1,85 & 46 & 0,00 & & 0,00 & & 0,75 & 88 \\
\hline $\begin{array}{l}\text { Lamiaceae } \\
\text { Aegiphila sellowiana Cham. } \\
\text { Vitex megapotamica (Spreng.) Moldenke }\end{array}$ & $\begin{array}{l}4,50 \\
3,02\end{array}$ & $\begin{array}{l}22 \\
32 \\
\end{array}$ & $\begin{array}{l}2,10 \\
2,56\end{array}$ & $\begin{array}{l}44 \\
34 \\
\end{array}$ & $\begin{array}{l}0,00 \\
0,00\end{array}$ & & $\begin{array}{l}2,34 \\
1,83 \\
\end{array}$ & $\begin{array}{l}36 \\
48 \\
\end{array}$ \\
\hline $\begin{array}{l}\text { Lauraceae } \\
\text { Cinnamomum glaziovii (Mez) Kosterm. } \\
\text { Cryptocarya aschersoniana Mez } \\
\text { Endlicheria paniculata (Spreng.) J.F.Macbr. }\end{array}$ & $\begin{array}{l}0,00 \\
0,55 \\
1,03\end{array}$ & $\begin{array}{l}79 \\
62\end{array}$ & $\begin{array}{l}0,00 \\
0,00 \\
3,26\end{array}$ & 24 & $\begin{array}{l}1,53 \\
2,67 \\
0,00\end{array}$ & $\begin{array}{l}53 \\
28\end{array}$ & $\begin{array}{l}0,50 \\
1,08 \\
1,38\end{array}$ & $\begin{array}{c}104 \\
83 \\
67\end{array}$ \\
\hline Nectandra megapotamica (Spreng.) Mez & 0,73 & 71 & 0,00 & & 0,75 & 80 & 0,49 & 106 \\
\hline Nectandra nitidula Nees & 0,00 & & 7,89 & 10 & 0,00 & & 2,61 & 28 \\
\hline Nectandra oppositifolia Nees & 2,92 & 33 & 2,36 & 36 & 3,44 & 27 & 2,82 & 24 \\
\hline Ocotea aciphylla (Nees) Mez & 0,70 & 72 & 1,35 & 58 & 1,74 & 46 & 1,27 & 70 \\
\hline Ocotea corymbosa (Meisn.) Mez & 2,85 & 34 & 2,22 & 40 & 8,09 & 11 & 4,23 & 19 \\
\hline Ocotea laxa (Nees) Mez & 0,00 & & 0,00 & & 0,87 & 62 & 0,26 & 122 \\
\hline Ocotea odorifera (Vell.) Rohwer & 9,83 & 5 & 4,95 & 15 & 9,78 & 8 & 8,18 & 5 \\
\hline Ocotea pulchella Mart. & 2,08 & 44 & 4,41 & 17 & 1,42 & 57 & 2,64 & 26 \\
\hline Persea rufotomentosa Nees \& Mart. ex Nees & 5,80 & 12 & 0,00 & & 0,00 & & 2,09 & 40 \\
\hline Persea willdenovii Kosterm. & 0,00 & & 1,87 & 48 & 0,00 & & 0,60 & 97 \\
\hline Loganiaceae & & & & & & & & \\
\hline Strychnos brasiliensis (Spreng.) Mart. & 0,00 & & 0,85 & 67 & 0,81 & 68 & 0,50 & 105 \\
\hline Lythraceae & & & & & & & & \\
\hline Lafoensia pacari A.St.-Hil. & 0,00 & & 2,16 & 41 & 0,00 & & 0,70 & 91 \\
\hline
\end{tabular}

Continua... 
TABELA 3: Continuação...

TABLE 3: Continued...

\begin{tabular}{|c|c|c|c|c|c|c|c|c|}
\hline \multirow{2}{*}{ Família / Espécie } & \multicolumn{8}{|c|}{ Setores } \\
\hline & \multicolumn{2}{|c|}{ A } & \multicolumn{2}{|c|}{$\mathrm{B}$} & \multicolumn{2}{|c|}{$\mathrm{C}$} & \multicolumn{2}{|c|}{ Total } \\
\hline Magnolia ovata (A.St.-Hil.) Sprengel & 0,00 & & 0,00 & & 6,10 & 13 & 1,96 & 44 \\
\hline \multicolumn{9}{|l|}{ Malpighiaceae } \\
\hline Byrsonima laxiflora Griseb. & 0,52 & 93 & 3,70 & 18 & 2,35 & 33 & 2,10 & 38 \\
\hline Byrsonima sericea DC. & 0,00 & & 2,12 & 43 & 0,00 & & 0,69 & 93 \\
\hline Byrsonima verbascifolia (L.) DC. & 0,51 & 95 & 0,00 & & 0,00 & & 0,21 & 154 \\
\hline Heteropterys byrsonimifolia A. Juss. & 7,09 & 10 & 2,77 & 30 & 0,86 & 64 & 3,82 & 20 \\
\hline \multicolumn{9}{|l|}{ Malvaceae } \\
\hline Eriotheca candolleana (K.Schum.) A.Robyns & 0,00 & & 0,00 & & 0,74 & 82 & 0,22 & 144 \\
\hline Luehea grandiflora Mart. \& Zucc. & 5,00 & 15 & 0,00 & & 0,00 & & 1,81 & 49 \\
\hline Pseudobombax longiflorum (Mart. \& Zucc.) A.Robyns & 0,00 & & 0,00 & & 1,57 & 50 & 0,51 & 103 \\
\hline \multicolumn{9}{|l|}{ Melastomataceae } \\
\hline Miconia argyrophylla DC. & 3,19 & 30 & 0,00 & & 0,00 & & 1,24 & 71 \\
\hline Miconia chartacea Triana & 3,55 & 26 & 0,00 & & 2,35 & 31 & 2,09 & 39 \\
\hline Miconia cinnamomifolia (DC.) Naudin & 0,00 & & 1,87 & 47 & 0,00 & & 0,56 & 99 \\
\hline Miconia pepericarpa DC. & 0,00 & & 0,00 & & 0,74 & 84 & 0,22 & 146 \\
\hline \multicolumn{9}{|l|}{ Meliaceae } \\
\hline Cabralea canjerana (Vell.) Mart. & 1,54 & 52 & 0,00 & & 0,74 & 86 & 0,79 & 87 \\
\hline Guarea macrophylla Vahl & 0,00 & & 0,00 & & 0,80 & 71 & 0,24 & 126 \\
\hline Trichilia catigua A.Juss. & 2,24 & 42 & 17,3 & 2 & 10,80 & 5 & 9,41 & 4 \\
\hline Trichilia pallens C.DC. & 0,61 & 76 & 0,00 & & 1,96 & 39 & 0,84 & 86 \\
\hline \multicolumn{9}{|l|}{ Memecylaceae } \\
\hline Mouriri glazioviana Cogn. & 0,73 & 70 & 3,10 & 27 & 0,76 & 78 & 1,42 & 65 \\
\hline \multicolumn{9}{|l|}{ Monimiaceae } \\
\hline Mollinedia argyrogyna Perkins & 1,51 & 54 & 0,99 & 63 & 0,00 & & 0,90 & 85 \\
\hline Mollinedia widgrenii A.DC & 1,19 & 56 & 0,00 & & 0,00 & & 0,47 & 109 \\
\hline \multicolumn{9}{|l|}{ Moraceae } \\
\hline Ficus adhatifolia Schott & 0,00 & & 0,00 & & 0,82 & 66 & 0,25 & 124 \\
\hline Ficus enormis (Mart.) Miq. & 0,00 & & 0,00 & & 0,94 & 61 & 0,28 & 120 \\
\hline Naucleopsis oblongifolia (Kuhlm.) Carauta & 0,00 & & 0,74 & 76 & 23,50 & 2 & 7,41 & 10 \\
\hline \multicolumn{9}{|l|}{ Myrsinaceae } \\
\hline Myrsine guianensis (Aubl.) Kuntze & 0,52 & 88 & 0,00 & & 0,00 & & 0,21 & 149 \\
\hline Myrsine lineata (Mez) Imkhan. & 0,52 & 89 & 0,00 & & 0,00 & & 0,21 & 150 \\
\hline Myrsine umbellata Mart. & 1,70 & 48 & 0,81 & 68 & 0,81 & 69 & 1,17 & 76 \\
\hline \multicolumn{9}{|l|}{ Myrtaceae } \\
\hline Blepharocalyx salicifolius (Kunth) O.Berg & 0,00 & & 0,00 & & 0,75 & 81 & 0,22 & 142 \\
\hline Calyptranthes brasiliensis Spreng. & 0,00 & & 0,00 & & 3,91 & 22 & 1,15 & 77 \\
\hline Calyptranthes clusiifolia O.Berg & 5,42 & 14 & 5,29 & 14 & 4,17 & 19 & 4,90 & 15 \\
\hline Campomanesia guazumifolia (Cambess.) O.Berg & 0,00 & & 0,00 & & 1,47 & 56 & 0,48 & 108 \\
\hline Eugenia dodonaeifolia Cambess. & 0,00 & & 0,00 & & 0,76 & 77 & 0,22 & 136 \\
\hline
\end{tabular}

Continua... 
TABELA 3: Continuação...

TABLE 3: Continued...

Família / Espécie

Eugenia florida DC.

Eugenia hyemalis Cambess.

Eugenia punicifolia (Kunth) DC.

Marlierea racemosa (Vell.) Kiaersk.

Myrcia guianensis (Aubl.) DC.

Myrcia hebepetala DC.

Myrcia pulchra Kiaersk.

Myrcia splendens (Sw.) DC.

Myrcia tomentosa (Aubl.) DC.

Myrcia venulosa DC.

Myrciaria floribunda (H.West ex Willd.) O.Berg

Siphoneugena densiflora O. Berg.

Siphoneugena reitzii D.Legrand

Siphoneugena widgreniana O.Berg

Nyctaginaceae

Guapira hirsuta (Choisy) Lundell

Guapira opposita (Vell.) Reitz

Ochnaceae

Ouratea semiserrata (Mart. \& Nees) Engl.

Opiliaceae

Agonandra excelsa Griseb.

\begin{tabular}{cccccccc}
\multicolumn{9}{c}{ Setores } \\
\multicolumn{2}{c}{ A } & \multicolumn{3}{c}{ B } & \multicolumn{2}{c}{ C } & \multicolumn{2}{c}{ Total } \\
1,71 & 47 & 0,73 & 78 & 1,49 & 55 & 1,34 & 68 \\
0,00 & & 0,76 & 74 & 0,00 & & 0,22 & 135 \\
2,74 & 35 & 0,00 & & 0,00 & & 1,11 & 80 \\
0,00 & & 0,00 & & 1,63 & 48 & 0,48 & 107 \\
0,00 & & 0,73 & 77 & 0,00 & & 0,22 & 147 \\
0,52 & 91 & 1,64 & 54 & 3,74 & 24 & 1,84 & 47 \\
0,00 & & 1,13 & 59 & 0,00 & & 0,35 & 116 \\
9,58 & 7 & 2,13 & 42 & 0,86 & 63 & 4,74 & 16 \\
1,69 & 49 & 0,00 & & 0,00 & & 0,68 & 94 \\
1,52 & 53 & 2,29 & 38 & 1,56 & 51 & 1,71 & 54 \\
0,00 & & 3,66 & 20 & 0,00 & & 1,09 & 82 \\
2,14 & 43 & 3,07 & 28 & 1,64 & 47 & 2,27 & 37 \\
0,00 & & 0,73 & 79 & 0,00 & & 0,21 & 151 \\
0,53 & 85 & 0,00 & & 0,00 & & 0,22 & 141 \\
\hline & & & & & & & \\
0,00 & & 0,00 & & 0,75 & 79 & 0,22 & 137 \\
0,00 & & 0,00 & & 0,74 & 83 & 0,22 & 145
\end{tabular}

\section{Phyllanthaceae}

Hieronyma alchorneoides Allemão

Polygonaceae

Coccoloba declinata (Vell.) Mart.

\section{Proteaceae}

Roupala montana Aubl.

Rosaceae

Prunus myrtifolia (L.) Urb.

Rubiaceae

Amaioua guianensis Aubl.

Cordiera concolor (Cham.) Kuntze

Faramea nigrescens Mart.

Ixora brevifolia Benth.

Psychotria vellosiana Benth.

0,62

$74 \quad 5,72$

$\begin{array}{llll}2,30 & 34 & 2,67 & 25\end{array}$

Randia armata (Sw.) DC.

$0,53 \quad 83 \quad 0,00$

0,00

$0,22 \quad 140$

Rudgea sp.

\section{Rutaceae}

Galipea jasminiflora (A.St.-Hil.) Engl. $0,67 \quad 73$

$73 \quad 1,72$

$1,72+2$

Metrodorea stipularis Mart.

0,00

$1,83 \quad 50 \quad 0,00$

$0,55 \quad 100$

$0,62 \quad 75 \quad 0,00$

0,00

$\begin{array}{llllllll}1,92 & 45 & 2,27 & 39 & 3,71 & 25 & 2,63 & 27\end{array}$

$\begin{array}{ccccccccc}4,83 & 17 & 1,51 & 56 & 8,95 & 9 & 5,14 & 14 \\ & 0,00 & & 2,32 & 37 & 0,00 & & 0,69 & 92 \\ & 0,00 & & 15,50 & 5 & 10,10 & 6 & 7,95 & 6 \\ & 3,07 & 31 & 16,76 & 4 & 3,93 & 21 & 7,55 & 9 \\ & 0,00 & & 0,77 & 73 & 0,00 & & 0,23 & 132 \\ & 0,54 & 81 & 0,00 & & 0,00 & & 0,22 & 139 \\ & 0,00 & & 0,00 & & 0,79 & 72 & 0,23 & 127 \\ & & & & & & & & \\ & 1,07 & 60 & 14,78 & 7 & 3,74 & 23 & 5,92 & 12 \\ & 1,39 & 55 & 6,55 & 11 & 0,00 & & 2,54 & 31\end{array}$

Continua... 
TABELA 3: Continuação...

TABLE 3: Continued...

\begin{tabular}{|c|c|c|c|c|c|c|c|c|}
\hline \multirow{3}{*}{$\begin{array}{l}\text { Família / Espécie } \\
\text { Zanthoxylum caribaeum Lam. }\end{array}$} & \multicolumn{8}{|c|}{ Setores } \\
\hline & \multicolumn{2}{|l|}{$\mathrm{A}$} & \multicolumn{2}{|c|}{$\mathrm{B}$} & \multicolumn{2}{|c|}{$\mathrm{C}$} & \multicolumn{2}{|c|}{ Total } \\
\hline & 0,87 & 64 & 0,00 & & 0,00 & & 0,32 & 118 \\
\hline Zanthoxylum monogynum A.St.-Hil. & 0,52 & 92 & 0,00 & & 0,00 & & 0,21 & 152 \\
\hline Zanthoxylum rhoifolium Lam. & 1,01 & 63 & 0,00 & & 0,00 & & 0,36 & 115 \\
\hline \multicolumn{9}{|l|}{ Salicaceae } \\
\hline Casearia decandra Jacq. & 4,61 & 19 & 3,59 & 21 & 1,62 & 49 & 3,47 & 22 \\
\hline Casearia lasiophylla Eichler & 1,64 & 51 & 0,00 & & 0,00 & & 0,67 & 95 \\
\hline Casearia obliqua Spreng. & 0,53 & 82 & 2,83 & 29 & 0,00 & & 1,06 & 84 \\
\hline Casearia sylvestris $\mathrm{Sw}$. & 4,57 & 20 & 1,92 & 46 & 0,00 & & 2,45 & 33 \\
\hline Xylosma prockia (Turcz.) Turcz. & 0,55 & 78 & 0,00 & & 0,00 & & 0,22 & 134 \\
\hline \multicolumn{9}{|l|}{ Sapindaceae } \\
\hline Cupania oblongifolia Mart. & 0,00 & & 0,00 & & 0,77 & 76 & 0,23 & 133 \\
\hline Cupania vernalis Cambess. & 18,15 & 3 & 1,13 & 60 & 0,74 & 88 & 7,86 & 8 \\
\hline Matayba guianensis Aubl. & 1,66 & 50 & 3,50 & 23 & 2,06 & 36 & 2,37 & 34 \\
\hline Matayba juglandifolia (Cambess.) Radlk. & 0,52 & 90 & 0,00 & & 1,77 & 44 & 0,75 & 89 \\
\hline \multicolumn{9}{|l|}{ Siparunaceae } \\
\hline Siparuna guianensis Aubl. & 0,82 & 67 & 1,76 & 51 & 2,57 & 29 & 1,59 & 59 \\
\hline \multicolumn{9}{|l|}{ Styracaceae } \\
\hline Styrax pohlii A. DC. & 0,80 & 69 & 0,00 & & 0,00 & & 0,32 & 117 \\
\hline \multicolumn{9}{|l|}{ Theaceae } \\
\hline Laplacea fruticosa (Schrad.) Kobuski & 0,00 & & 0,00 & & 0,78 & 75 & 0,23 & 129 \\
\hline \multicolumn{9}{|l|}{ Thymelaeaceae } \\
\hline Daphnopsis brasiliensis Mart. \& Zucc. & 0,00 & & 0,00 & & 1,49 & 54 & 0,43 & 113 \\
\hline \multicolumn{9}{|l|}{ Vochysiaceae } \\
\hline Qualea cordata (Mart.) Spreng. & 0,53 & 86 & 0,00 & & 0,00 & & 0,22 & 143 \\
\hline Qualea grandiflora Mart. & 0,00 & & 0,90 & 66 & 0,00 & & 0,27 & 121 \\
\hline Vochysia magnifica Warm. & 0,00 & & 4,90 & 16 & 0,00 & & 1,60 & 58 \\
\hline Vochysia tucanorum Mart. & 0,59 & 77 & 3,67 & 19 & 0,00 & & 1,44 & 63 \\
\hline
\end{tabular}

2008; CARVALHO et al., 2009).

As duas espécies com maiores VI no setor A, Copaifera langsdorffii e Cordia sellowiana, são espécies de crescimento lento (CHAGAS et al., 2001) e, com mais de 40 anos do corte e queima, destacam-se na estrutura da comunidade arbórea deste setor. Outras espécies, características de ambientes mais secos, também apresentaram altos VI, podendo ter sido favorecidas pelo corte raso. Sabe-se que a proximidade de áreas abertas influencia na colonização e estabelecimento de espécies que necessitam de menor disponibilidade de água e que toleram maior intensidade de luz (MACDOUGALL; KELLMAN, 1992; TABARELLI; CARDOSO; GASCON, 2004). Isto explica a importância de Machaerium villosum, característica de solos com drenagem acentuada (MACHADO et al., 2008). O mesmo é válido para Tapirira obtusa, Heteropterys byrsonimifolia e Luehea grandiflora, já que a transição florestacerrado, típica na região, é rica destas espécies, que também são características de ambientes de borda (OLIVEIRA FILHO; RATTER, 1995; CARVALHO et al., 2000; PRADO JÚNIOR et al., 2010).

O contrário é válido para o setor $\mathrm{C}$, que se localiza em uma ravina com maior suprimento de água e menor exposição a ventos. Muitas das espécies importantes neste setor são facilmente encontradas em florestas ribeirinhas, sendo consideradas típicas de ambientes ripários (CARVALHO et al., 2000; ALVES; MARTINS; SANTOS, 2004; TEIXEIRA et al., 2008). Destas, podem ser destacadas Euterpe edulis, Calophyllum brasiliense e Magnolia ovata; além de outras espécies afeitas à umidade, contudo, não tão características de ambientes úmidos, como Naucleopsis oblongifolia e Copaifera trapezifolia. Espécies mais generalistas como Protium widgrenii 


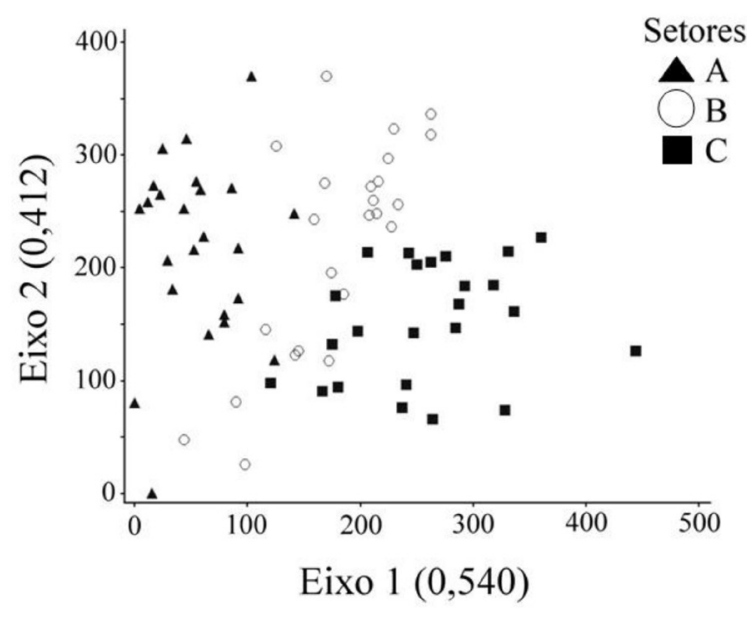

FIGURA 2: Diagrama de ordenação para a Análise de Correspondência Retificada (DCA) realizada para as parcelas de três setores de um remanescente florestal situado no Município de Itumirim - MG Variância total dos dados das espécies $($ Inércia $)=11,38 \%$.

FIGURE 2: Ordination diagram for Detrended Correspondence Analysis for 24 plots in three sectors of a forest fragment located in Itumirim, Minas Gerais state, Brazil. Total variance of species $($ Inertia $)=11.38 \%$.

e Ocotea corymbosa também apresentaram altos VI, o que pode ser considerado como consequência da heterogeneidade ambiental da área (CARVALHO et al., 1995; SCIPIONI et al., 2012). Já o setor B, por apresentar espécies como Trichilia catigua, Cheiloclinium cognatum e Ixora brevifolia como possuidoras de altos VI, se diferencia do setor A por estas não serem espécies marcadamente de estágios sucessionais iniciais e do setor $\mathrm{C}$ por não possuírem caráter ripário.

Corroborando estes dados, a DCA também faz distinção entre os setores. $\mathrm{O}$ diagrama mostra o setor A distinto do setor $\mathrm{C}$ e o setor $\mathrm{B}$ em uma situação de transição entre os dois (Figura 2). Das parcelas do setor A, 19 apresentaram tendência de agrupamento à esquerda do diagrama, no início do eixo 1 (autovalor $=0,540$ ) e na metade do eixo 2 (autovalor $=0,412$ ). As parcelas do setor C apresentaram-se mais distribuídas, porém, pareceram seguir um padrão diagonal no sentido crescente dos eixos 1 e 2 , com maior amplitude no primeiro eixo, que apresenta maior parcela de explicação dos dados. Já as parcelas do setor B apresentaram um padrão aparentemente semelhante ao apresentado pelo setor $\mathrm{C}$, porém, com maior amplitude no eixo 2. O autovalor do eixo 1 maior que 0,5 indica que há substituição de espécies entre os extremos do eixo (BRAAK, 1995), o que contribui para distinguir ainda mais os setores A e C, situados nestes extremos, e evidenciar o gradiente de espécies entre ambos, com o setor B intermediário a eles, no entanto, mais próximo do setor $\mathrm{C}$, já que a maioria de suas parcelas se agrupa com as deste setor, principalmente considerando o primeiro eixo.

\section{CONCLUSÕES}

De acordo com os dados obtidos neste trabalho, pode-se concluir que a vegetação arbórea dos três setores avaliados possui parâmetros de diversidade, estrutura e composição distintos, o que em muito se deve aos seus respectivos históricos de perturbações. Os parâmetros observados no setor A condizem com o tipo de vegetação observado em áreas de floresta em regeneração e que podem ser devidos ao corte e queima da vegetação estabelecida anteriormente; enquanto o setor $\mathrm{C}$ apresentou parâmetros que são observados com frequência em florestas livres de perturbações. Já o setor B parece estar em uma situação intermediária, devido a uma aparente interferência do pisoteio do gado sobre a vegetação. Entretanto, ressalta-se que esta perturbação só poderá ter seu efeito verificado em estudos de acompanhamento regular.

\section{REFERÊNCIAS BIBLIOGRÁFICAS}

ALVES, L. F.; MARTINS, F. R.; SANTOS, F. A. M. Allometry of a neotropical palm, Euterpe edulis Mart. Acta Botanica Brasilica, São Paulo, v. 18, n. 2, p. 369-374, abr./jun. 2004.

APG. Angiosperm Phylogeny Group. An update of the Angiosperm Phylogeny Group classification for the orders and families of flowering plants: APG II. Botanical Journal of the Linnean Society, London, v. 141, n. 4, p. 399-436, mar. 2003.

BOTREL, R. T. et al. Influência do solo e topografia sobre as variações da composição florística e estrutural da comunidade arbóreo-arbustiva de uma floresta estacional semidecidual em Ingaí, MG. Revista Brasileira de Botânica, São Paulo, v. 25, n. 2, p. 195-213, mar./abr. 2002. 
BRAAK, C. J. F. The analysis of vegetation environment relationships by canonical correspondence analysis. Vegetatio, The Hage, v. 69, n. 3, p. 69-77, apr. 1987.

Ordination. In: JONGMAN, R. H. G.; BRAAK, C. J. F.; TONGEREN, O. F. R. V. (Ed.). Data analysis in community and landscape ecology. Cambridge: University Press Cambridge, 1995. p. 91-173.

BRASIL. Ministério da Agricultura e da Reforma Agrária. Departamento Nacional de Meteorologia. Normais climatológicas: 1961-1990. Brasília, DF, 1992. $84 \mathrm{p}$.

BROWER, J. E.; ZAR, J. H. Field and laboratory methods for general ecology. 2. ed. Dubuque: W.M.C. Brow, 1984. 226 p.

BROWN, S.; LUGO, A. E. Tropical secondary forests. Journal of Tropical Ecology, Cambridge, v. 6, n. 1, p. 1-32, jan./feb. 1990.

CANTARELLO, E. et al. Simulating the potential for ecological restoration of dryland forests in Mexico under different disturbance regimes. Ecological Modelling, Amsterdam, v. 222, n. 5, p. 1112-1128, mar. 2011.

CARVALHO, D. A. et al. Estrutura fitossociológica de mata ripária do alto Rio Grande, Bom Sucesso, estado de Minas Gerais. Revista Brasileira de Botânica, São Paulo, v. 18, n. 1, p. 39-49, jan./fev. 1995.

Florística e estrutura da vegetação arbórea de um fragmento de floresta semidecidual às margens do reservatório da Usina Hidrelétrica Dona Rita (Itambé do Mato Dentro, MG). Acta Botanica Brasilica, São Paulo, v. 14, n. 1, p. 37-55, jan./mar. 2000.

CARVALHO, F. A.; NASCIMENTO, M. T. Estrutura diamétrica da comunidade e das principais populações arbóreas de um remanescente de floresta atlântica submontana (Silva Jardim-RJ, Brasil). Revista Árvore, Viçosa, v. 33, n. 2, p. 327-337, mar./abr. 2009.

CARVALHO, L. C. S. et al. Estrutura temporal de sete populações em três fragmentos florestais no Alto Rio Grande, Minas Gerais. Cerne, Lavras, v. 15, n. 1, p. 58-66, jan./mar. 2009.

CHAGAS, R. K. et al. Dinâmica de populações arbóreas em um fragmento de floresta estacional semidecidual montana em Lavras, Minas Gerais. Revista Árvore, Viçosa, v. 25, n. 1, p. 39-57, jan./fev. 2001.

CONNELL, J. H.; SLATYER, R. O. Mechanisms of succession in natural communities and their role in community stability and organization. The
American Naturalist, Chicago, v. 111, n. 982, p. 1119-1140, nov./dec. 1977.

DEAN, W. A ferro e fogo: a história e a devastação da Mata Atlântica brasileira. São Paulo: Companhia das Letras, 1996. 484 p.

EMBRAPA. Empresa Brasileira de Pesquisa Agropecuária. Sistema brasileiro de classificação de solos. 2. ed. Rio de Janeiro: Embrapa, 2006. $306 \mathrm{p}$.

FINOTTI, R. et al. Variação na estrutura diamétrica, composição florística e características sucessionais de fragmentos florestais da bacia do rio Guapiaçu (Guapimirim/Cachoeiras de Macacu, RJ, Brasil). Acta Botanica Brasilica, São Paulo, v. 26, n. 2, p. 464-475, abr./jun. 2012.

GUILHERME, F. A. G. et al. Soil profile, relief features and their relation to structure and distribution of Brazilian Atlantic Rain forest trees. Scientia Agricola, São Paulo, v. 69, n. 1, p. 61-69, jan./fev. 2012.

HORNER, G. J. et al. Forest structure, flooding and grazing predict understorey composition of floodplain forests in southeastern Australia. Forest Ecology and Management, Amsterdam, v. 286, p. 148-158, dec. 2012.

IMAI, N. et al. Effects of selective logging on tree species diversity and composition of Bornean tropical rain forests at different spatial scales. Plant Ecology, Dordrecht, v. 213, n. 9, p. 1413-1424, sep. 2012.

JONES, M. M.; SZYSKA, B.; KESSLER, M. Microhabitat partitioning promotes plant diversity in a tropical montane forest. Global Ecology and Biogeography, Malden, v. 20, n. 4, p. 558-569, jul. 2011.

LAURANCE, W. F. et al. The fate of Amazonian forest fragments: a 32-year investigation. Biological Conservation, Amsterdam, v. 144, n. 1, p. 56-67, jan. 2011.

MACDOUGALL, A.; KELLMAN, M. The understorey light regime and patterns of tree seedlings in tropical riparian forest patches. Journal of Biogeography, Oxford, v. 19, n. 5, p. 667-675, sep./oct. 1992.

MACHADO, E. L. M. et al. Efeitos do substrato, bordas e proximidade espacial na estrutura da comunidade arbórea de um fragmento florestal em Lavras, MG. Revista Brasileira de Botânica, São Paulo, v. 31, n. 2, p. 287-302, abr./jun. 2008.

MAGNAGO, L. F. S. et al. Variações estruturais e características edáficas em diferentes estádios sucessionais de floresta ciliar em tabuleiro, ES. 
Revista Árvore, Viçosa, v. 35, n. 3, p. 445-456, mai./jun. 2011.

MARTINEZ-GARZA, C.; HOWE, H. F. Restoring tropical diversity: beating the time tax on species loss. Journal of Applied Ecology, London, v. 40, n. 3, p. 423-429, mar. 2003.

MUELLER-DOMBOIS, D.; ELLENBERG, $\mathrm{H}$. Aims and methods of vegetation ecology. New York: Wiley, 1974. 574 p.

NUNES, Y. R. F. et al. Variações da fisionomia, diversidade e composição de guildas da comunidade arbórea em um fragmento de floresta semidecidual em Lavras, MG. Acta Botanica Brasilica, São Paulo, v. 17, n. 2, p. 213-229, abr.jun. 2003.

OLIVEIRA FILHO, A. T. et al. Diversity and structure of the tree community of a patch of tropical secondary forest of the Brazilian Atlantic Forest Domain 15 and 40 years after logging. Revista Brasileira de Botânica, São Paulo, v. 27, n. 4, p. 685-701, out./dez. 2004.

MELLO, J. M.; SCOLFORO, J. R. S. Effects of past disturbance and edges on tree community structure and dynamics within a fragment of tropical semideciduous forest in south-eastern Brazil over a five-year period: 1987-1992. Plant Ecology, Dordrecht, v. 131, n. 1, p. 45-66, jan./feb. 1997.

RATTER, J. A. A study of the origin of central Brazilian forests by the analysis of plant species distribution patterns. Edinburgh Journal of Botany, Edinburgh, v. 52, n. 2, p. 141-194, mar./abr. 1995.

SCOLFORO, J.R. S.; MELLO, J. M.de. Composição florística e estrutura de um remanescente de floresta semidecidual montana em Lavras, MG. Revista Brasileira de Botânica, São Paulo, v. 17, n. 2, p. 167-182, abr./jun. 1994.

OMEJA, P. A. et al. Intensive tree planting facilitates tropical forest biodiversity and biomass accumulation in Kibale National Park, Uganda. Forest Ecology and Management, Amsterdam, v. 261, n. 3, p. 703-709, feb. 2011.

PALMER, M. W. Estimating species richness: the second-order jackknife estimator reconsidered. Ecology, Durham, v. 72, n. 4, p. 1512-1513, aug. 1991.

PEREIRA, J. A. A.; OLIVEIRA FILHO, A. T.; LEMOS FILHO, J. P. Environmental heterogeneity and disturbance by humans control much of the tree species diversity of fragments of tropical montane seasonal forests in SE Brazil. Biodiversity and Conservation, Amsterdam, v. 16, n. 6, p. 1761-1784, dec. 2007.
PRADO JÚNIOR, J. A. et al. Estrutura da comunidade arbórea em um fragmento de floresta estacional semidecidual localizada na reserva legal da Fazenda Irara, Uberlândia, MG. Bioscience Journal, Uberlândia, v. 26, n. 4, p. 638-647, jul./ago. 2010.

RODRIGUES, L. A. et al. Florística e estrutura da comunidade arbórea de um fragmento florestal em Luminárias, MG. Acta Botanica Brasilica, São Paulo, v. 17, n. 1, p. 71-87, jan./mar. 2003.

SCIPIONI, M. C. et al. Análise fitossociológica de um fragmento de floresta estacional em uma catena de solos no Morro do Cerrito, Santa Maria, RS. Ciência Florestal, Santa Maria, v. 22, n. 3, p. 457-466, jul./set., 2012.

TABARELLI, M.; CARDOSO, J. M.; GASCON, C. Forest fragmentation synergisms and the impoverishment of neotropical forests. Biodiversity and Conservation, Amsterdam, v. 13, n. 7, p. 1419-1425, jul. 2004.

TABARELLI, M.; MANTOVANI, W. A regeneração de uma Floresta Tropical Montana após corte e queima (São Paulo-Brasil). Revista Brasileira de Biologia, São Paulo, v. 59, n. 2, p. 239-250, abr./jun. 1999.

TEIXEIRA, A. P. et al. Tree species composition and environmental relationships in a Neotropical swamp forest in Southeastern Brazil. Wetlands Ecology and Management, Amsterdam, v. 16, n. 6, p. 451-461, dec. 2008.

TERBORGH, J. Diversity and the Tropical Rain Forest. New York: Scientific American Library, 1992. $242 \mathrm{p}$.

UHL, C.; MURPHY, P. G. Composition, structure, and regeneration of a tierra firme forest in the Amazon Basin of Venezuela. Tropical Ecology, Varanasi, v. 22, n. 2, p. 219-237, jul. 1981.

VELOSO, H. P.; RANGEL FILHO, A. L. R.; LIMA, J. C. A. Classificação da vegetação brasileira adaptada a um sistema universal. Rio de Janeiro: IBGE, 1991. 124 p.

VILELA, M. S. A formação histórica dos Campos de Sant'Ana das Lavras do Funil. Lavras: Indi, 2007. 450 p.

WILLIAMS-LINERA, G.; LOREA, F. Tree species diversity driven by environmental and anthropogenic factors in tropical dry forest fragments of central Veracruz, Mexico. Biodiversity and Conservation, Amsterdam, v. 18, n. 12, p. 3269-3293, nov. 2009. ZAR, J. H. Biostatistical analysis. New Jersey: Prentice-Hall, 1996. 718 p. 